\title{
Gli intellettuali del Risorgimento a Genova, tra spirito repubblicano, sovranismo e federalismo. Il pensiero e le arti
}

Les intellectuels du Risorgimento à Gênes, entre esprit républicain, souveraineté et fédéralisme. La pensée et les arts

The Intellectuals of the Risorgimento in Genoa, between Republican Spirit, Sovereignty and Federalism. Thought and the Arts

Maurizia Migliorini, Francesca Gastaldo e Chiara Capobianco

(2) OpenEdition

Journals

Edizione digitale

URL: https://journals.openedition.org/cei/10476

DOI: $10.4000 /$ cei. 10476

ISSN: 2260-779X

Editore

UGA Éditions/Université Grenoble Alpes

Edizione cartacea

ISBN: 978-2-37747-342-7

ISSN: 1770-9571

Notizia bibliografica digitale

Maurizia Migliorini, Francesca Gastaldo e Chiara Capobianco, «Gli intellettuali del Risorgimento a Genova, tra spirito repubblicano, sovranismo e federalismo. II pensiero e le arti», Cahiers d'études italiennes [Online], 34 | 2022, online dal 03 mars 2022, consultato il 04 mars 2022. URL: http:// journals.openedition.org/cei/10476 ; DOI: https://doi.org/10.4000/cei.10476

Questo documento è stato generato automaticamente il 4 mars 2022.

(c) ELLUG 


\section{Gli intellettuali del Risorgimento a Genova, tra spirito repubblicano, sovranismo e federalismo. Il pensiero e le arti}

Les intellectuels du Risorgimento à Gênes, entre esprit républicain, souveraineté et fédéralisme. La pensée et les arts

The Intellectuals of the Risorgimento in Genoa, between Republican Spirit, Sovereignty and Federalism. Thought and the Arts

Maurizia Migliorini, Francesca Gastaldo e Chiara Capobianco

\section{Il contesto culturale}

1 È emozionante in questo tempo di sovranismo e individualismo tornare indietro di due secoli per riabbracciare lo spirito civico a Genova, perennemente in bilico tra repubblica e monarchia, federalismo e indipendentismo, ma sempre alla generosa ricerca delle radici della propria comunità.

2 Questo ci racconta una città repubblicana e mazziniana, terra di spiriti liberi, costretta all'indomani del Congresso di Vienna, dopo l'ubriacatura napoleonica, a sottomettersi alla dinastia sabauda, rinunciando alla propria identità, prima sotto Carlo Emanuele I e poi sotto Carlo Felice, denominato dai genovesi «Carlo Feroce».

3 Durante il Congresso di Vienna e precisamente la notte del 25 dicembre del $1814 \mathrm{fu}$ decisa la fine della Repubblica di Genova e l'annessione mai digerita al regno sabaudo. Il prefetto governativo marchese Gerolamo Serra viene così ricordato:

Non appena in Genova penetrò la notizia di queste finali decisioni, il popolo avrebbe voluto levarsi coll'armi, e solo con esse alla mano cedere all'imperio della forza. Ma Gerolamo Serra, che al riverito nome congiungeva la prontezza e l'efficacia del dire,

dopo di avere dimostrato l'intero voto d'Europa assolutamente contrario 
all'esistenza dello Stato genovese, ebbe il contento di risparmiare all'amatissima patria una nuova e tremenda sciagura. coinvolti nelle vicende politiche intellettuali e civili della città, autore di un fortunato libro sulla vita privata dei genovesi nel quale racconta gli aspetti più curiosi e inediti dei genovesi dal Medioevo fino a tutto il Rinascimento, desunte dalle sue fitte ricerche negli archivi storici cittadini.

5 La presenza di Giuseppe Mazzini aveva segnato pesantemente lo spirito repubblicano genovese. Una città oligarchica, ma senza forti figure aristocratiche di riferimento, fatta una sporadica eccezione per Andrea Doria, dà i natali all'uomo più scomodo e carismatico del Risorgimento italiano.

6 La straordinaria agiografia mazziniana di Jesse vedova Mario, una delle tante eroine rivoluzionarie e repubblicane del primo Ottocento genovese ci consegna un eroe quasi cristologico, illuminato fin dall'infanzia da una sorta di spirito divino che lo guidò, attraverso mille traversie, alla grandezza e all'unità del paese ${ }^{2}$.

7 Jessie Jane Meriton White, coniugata Mario, nata a Gosport nel 1832 da ricchi armatori, dopo essere stata imprigionata quattro mesi a Genova per il fallimento dell'impresa di Carlo Pisacane, sposa a Genova Alberto Mario e diviene una prima reporter del Risorgimento italiano, lanciando la tecnica del reportage giornalistico che applica a piene mani nella sua straordinaria biografia mazziniana, che diviene la prima storia del Risorgimento italiano.

8 Jessie, ricordata come miss Uragano per il suo giornalismo d'assalto e le sue idee incendiarie, incarna le prime manifestazioni della sinistra storica italiana pre- e postunitaria intrisa di spirito repubblicano, di liberalismo e di idee ugualitarie. Partecipò in veste di infermiera a quattro campagne di Giuseppe Garibaldi; fece ricerche sulle condizioni di vita nei quartieri più poveri di Napoli e dei minatori siciliani, anticipando Matilde Serao.

(1857, prima dell'ultimo e definitivo esilio, tentò di organizzare in città l'insurrezione di Genova e Livorno, mentre Carlo Pisacane fece partire, con l'aiuto dell'armatore Raffaele Rubattino, la sfortunata spedizione di Sapri per la liberazione del Regno delle due Sicilie, ricordata nei celeberrimi versi di Luigi Mercantini.

10 Ma ben prima delle vicende di Giuseppe Mazzini, Giovanni Ruffini e Carlo Pisacane, giovani intellettuali meno noti e più timidamente inseriti in contesti cittadini, diedero vita al nuovo giornalismo democratico. Molti di loro collaborarono con «L'Espero», battagliero foglio politico- letterario (1841-1846) soppresso dalla polizia asburgica. Erano questi Emanuele Celesia ${ }^{3}$, letterato, Federico Alizeri', storico dell'arte ante litteram, Michele Giuseppe Canale ${ }^{5}$, storico, Giovanni Battista Cevasco, scultore, amici e compagni di tante battaglie che, nel ricordo di una combattiva gioventù mazziniana $\mathrm{e}$ di alcune bellicose rivolte approdarono infine, molto più pacatamente, a un impegno istituzionale non privo di ricordi di antiche lotte cittadine.

11 Le arti, la musica e il teatro divennero per loro uno straordinario collante politico che ripercorse tutte le tappe del movimentismo mazziniano nelle insurrezioni del 1948, del 1849, e infine in quella fallita del 1857.

Emanuele Celesia, come del resto l'amico Federico Alizeri nel 1849, scrisse il resoconto della rivoluzione genovese del $1848^{6}$ indicando i fatti salienti di quella insurrezione che furono, nell'ordine: la cacciata dei padri Gesuiti che sostenevano il governo asburgico, il 
mutuo soccorso cittadino, la centralità del nuovo teatro Carlo Felice, con feste inni e gran sventolare di bandiere tricolori, a sostegno della lotta contro i Borboni, la festa della Costituzione con il canto del Te Deum nella Cattedrale di San Lorenzo.

Il fatto che persino Anita Garibaldi fosse venuta a Genova con i tre figli nel marzo 1948 è un segno dell'importanza della rivolta genovese.

Verso la fine dell'anno il movimento si concluse con un nulla di fatto che volgerà verso il triumvirato formato da Giuseppe Avezzana, Davide Morchio e Costantino Reta, uomini dell'istituzione che passano dalla parte della rivolta popolare, che proseguirà nella celeberrima insurrezione del 1849, descritta, per incarico del Comune, da Federico Alizeri.

15 Il testo di Federico Alizeri ${ }^{7}$, ricco e coinvolgente, con allegate le disposizioni reali e ministeriali e la lotta dei triumviri, narra la rivolta genovese contro l'armistizio del 1849, che coinvolse cittadini, nobili e borghesia, e soprattutto gli intellettuali di formazione mazziniana come Michele Giuseppe Canale, Emanuele Celesia, e altri intellettuali come la famiglia d'Aste e il marchese Giancarlo Di Negro.

Le rivolte di Genova del 1849 avvenute a seguito dell'armistizio, firmato il 9 agosto 1848 a Vigevano dal generale piemontese Carlo Canera di Salasco e dal generale austriaco von Hess, misero fine alla prima fase della Prima guerra d'indipendenza, con una seconda restaurazione politica e territoriale che ripropose il sovranismo e i confini imposti nel 1815 dal Congresso di Vienna.

17 Più famosa della rivolta genovese, raccontata da Alizeri, fu quella di Venezia, dell'agosto 1849 , fallita miseramente anche per l'epidemia di colera che aveva indebolito la città. Arnaldo Fusinato fu uno dei poeti che descrisse la caduta di Venezia con la famosissima ode a Venezia conclusa con le celeberrime strofe «il morbo infuria il pan ci manca, sul ponte sventola bandiera bianca!».

18 Arnaldo Fusinato, poeta veneto, compare, alla fine delle vicende politiche del 1848, di passaggio a Genova, insieme a molti altri intellettuali del Risorgimento nel Diario di Elena d'Aste ${ }^{8}$, grazioso quaderno manoscritto femminile della figlia del professore di calligrafia e scrittore di testi teatrali Ippolito, amico del marchese Di Negro e intellettuale liberale che partecipò a tutti i moti del 1848-1849, ma che visse anche un momento di ritorno all'ordine in monarchia dopo l'Unità d'Italia nel 1870.

Sull'elegante quaderno della figlia compaiono, tutti rigorosamente manoscritti, pensieri e versi poetici inediti di grandissimi protagonisti dell'Unità d'Italia da Giuseppe Mazzini, a Nicolò Tommaseo, fino ad Aleardo Aleardi, che passò dalle lotte mazziniane al Senato del Regno d'Italia. Tra loro ci fu anche chi non rinunciò alle lotte e all'esilio come Francesco Dall'Ongaro, ex sacerdote convertito alle lotte repubblicane o chi, uomo della sinistra storica, si inserì dopo l'unità del Paese nelle istituzioni pubbliche. Nel diario di Elena troviamo anche un pensiero di Atto Vannucci del 1852, ancora esule, ma che al suo rientro in Italia si schierò su posizioni filogovernative $\mathrm{e}$ monarchiche, pur rimanendo profondamente anticlericale.

In quel diario viene pubblicato anche, per la prima volta il 26 agosto del 1848, dalla mano stessa di Goffredo Mameli, un famoso Inno militare.

21 Il testo, vergato nel pieno delle guerre di indipendenza, compare qui manoscritto (foto 1a e 1b) nell'album della giovane Elena. 
Foto 1a. - Goffredo Mameli, Versione autografa dell'Inno Militare, 1848, Album di Elena d'Aste.

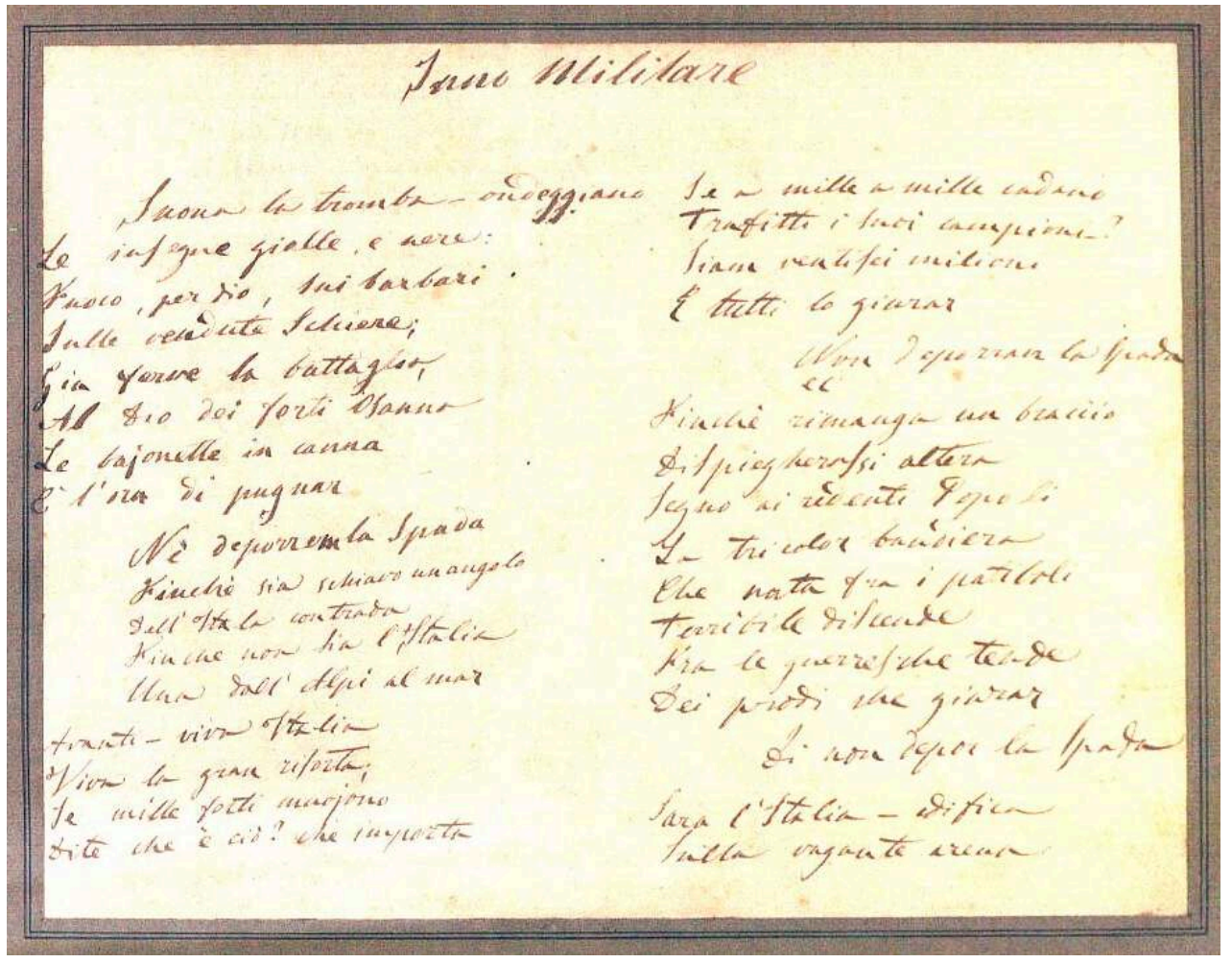

Biblioteca Civica Berio, Sezione Conservazione.

Foto 1b. - Goffredo Mameli, Versione autografa dell'Inno Militare, 1848, Album di Elena d'Aste.

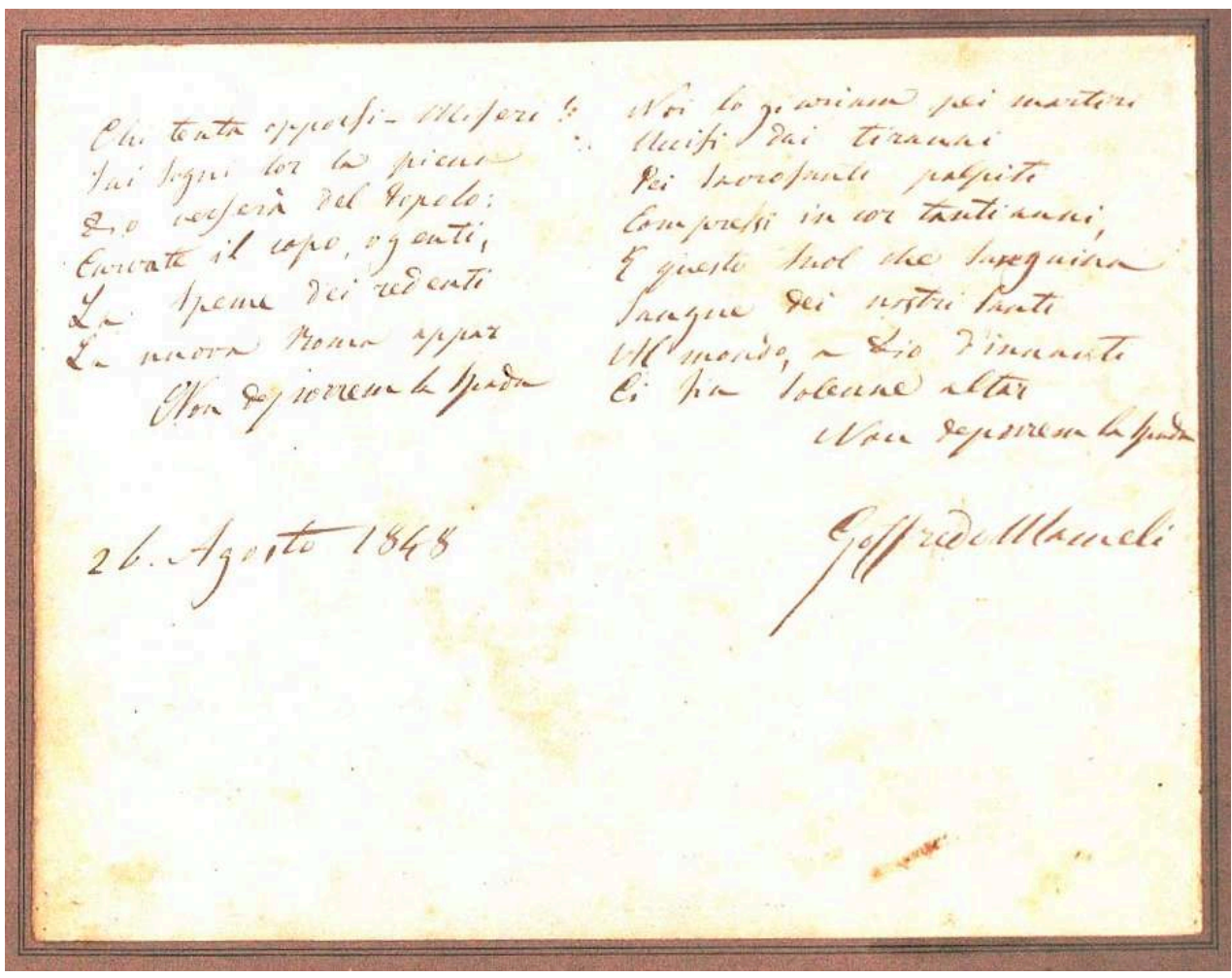

Biblioteca Civica Berio, Sezione Conservazione. 
Suona la tromba. Ondeggiano

le insegne gialle e nere.

Fuoco, per Dio, sui barbari, sulle vendute schiere.

Già ferve la battaglia

al Dio dei forti, Osanna,

le bajonette in canna

è l'ora di pugnar.

Non deporrem la spada

finché sia schiavo un angolo

dell'Itala contrada,

finché non sia l'Italia

una dall'Alpi al mar.

Avanti. Viva Italia,

viva la gran risorta:

se mille forti muoiono

dite, che è ciò? Che importa?

Se a mille a mille cadono,

trafitti, i suoi campioni?

siam ventisei milioni

e tutti lo giurar.

(Non deporrem la spada...)

Finché rimanga un braccio

dispiegherassi altera

segno ai ridenti popoli

la tricolor bandiera

che, nata fra i patiboli, terribile discende

tra le guerresche tende

dei prodi che giurar.

(Non deporrem la spada...)

Sarà l'Italia. Edifica

sulla vagante arena.

Chi tenta opporsi? Miseri!

Dei sogni lor la piena

Dio veglierà del popolo.

Curvate il capo, o genti,

la speme dei redenti

la nuova Roma appar.

(Non deporrem la spada...)

Noi lo giuriam pei martiri

uccisi dai tiranni

pei sacrosanti palpiti

compressi in cor tanti anni

e questo suol che sanguina

sangue dei nostri santi.

Al mondo, a Dio dinnanti

ci sia solenne altar.

(Non deporrem la spada...)

22 Scritto il 26 agosto 1848, come protesta contro l'armistizio Salasco, che segnava la capitolazione del Piemonte, l'Inno militare fu in seguito musicato da Giuseppe Verdi. Scritto, dunque, un anno dopo l'Inno Nazionale italiano Fratelli d'Italia, che al contrario era stato messo in musica da Michele Novaro, l'Inno militare era stato fortemente voluto da Mazzini, ma poi rifiutato dallo stesso per il troppo esplicito riferimento ai colori della bandiera dell'Austria. 

repubblicani, in un contesto di civile sopportazione della dinastia sabauda, ma anche nel dignitoso mantenimento delle sue radici. Fu quanto la città manifestò, nel marzo 1872, nel trionfale ritorno della salma di Giuseppe Mazzini a Genova quando, dopo la sua morte a Pisa, l'apostolo della rivoluzione repubblicana fu riportato nella sua città. Il cimitero monumentale di Staglieno lo accolse in un tempietto neoclassico costruito per lui dall'architetto mazziniano Gaetano Vittorio Grasso che si fece seppellire di fronte a lui nel boschetto irregolare della necropoli, dove trovarono riposo, ad uno ad uno, nel tempo, i garibaldini dell'impresa dei Mille e molti dei mazziniani ricordati in questo contesto, come Federico Alizeri ed Emanuele Celesia.

Per introdurre le riflessioni che seguono, si può dire che, a cominciare dallo stesso Mazzini, furono proprio gli intellettuali, con la musica e le arti, a portare avanti le più raffinate strategie rivoluzionarie, ma anche le politiche di adattamento e di adeguamento alla monarchia sabauda che seguirono, in attesa della repubblica che in Italia si realizzò solo un secolo dopo le grandi rivoluzioni del 1848. E tra i grandi mazziniani fu soprattutto lo scultore Giovanni Battista Cevasco a coniugare le istanze repubblicane con un rinnovato spirito civico postunitario, sia nell'ambito della grande 
committenza cittadina per la Necropoli di Staglieno, sia per il suo quotidiano impegno di assessore alla Giunta Comunale della città. (Maurizia Migliorini)

\section{Giovanni Battista Cevasco, scultore e politico genovese}

Il periodo di dominazione francese a Genova inizia nel 1797 e si chiude soltanto con la definitiva sconfitta di Napoleone nel 1815. La città viene assorbita dalla potenza francese e perde il suo spirito indipendente e ribelle contro le dominazioni straniere. L'assetto urbanistico del territorio genovese risente di numerose innovazioni e ampliamenti architettonici. Nel 1804 Napoleone emana L'Editto di Saint Cloud, secondo il quale i cimiteri devono essere posti al di fuori delle mura urbane, seguire criteri igienico-sanitari precisi e conferire dignità ai deceduti. A seguito di questo editto si sviluppa il cimitero monumentale di Staglieno, in cui vengono elevate importanti opere di scultura funeraria di impostazione romantica e realistica. Qui lavorano numerosi scultori, tra i quali Giovanni Battista Cevasco, politico ed artista ligure, che si forma presso l'Accademia Ligustica di Belle Arti di Genova; egli stringe sodalizi artistici e politici con molti personaggi illustri, non solo genovesi, che gli permettono di realizzare numerose opere d'arte su tutto il territorio italiano. L'artista, come si evince dai suoi scambi epistolari con importanti personalità politiche dell'epoca tra cui Vincenzo Ricci, Nino Bixio e Giuseppe Garibaldi, è stato un attivo sostenitore del Risorgimento, come è testimoniato dal fatto che la sua carriera politica è parallela a quella artistica.

Negli anni dal 1848 al 1878 egli ricopre la carica di consigliere comunale genovese ed è anche attivo come scultore nel cimitero di Staglieno, dove realizzerà numerose statue $\mathrm{e}$ tombe di un buon livello artistico. Sempre negli stessi anni entra in contatto con la famiglia Durazzo-Pallavicini con cui collabora realizzando numerose sculture oggi collocate nella Villa Durazzo-Pallavicini. Stringe anche rapporti di amicizia con la marchesa Maria Brignole-Sale, moglie di Raffaele De Ferrari, che fece la sua fortuna economica a Parigi, dove visse a lungo. Maria Brignole Sale, erede di una delle più importanti famiglie della nobiltà genovese, fu benefattrice locale, ma sia lei che il marito e il figlio vissero a lungo a Parigi. La marchesa e il marito avevano fondato varie opere di beneficenza, tra cui l'ospedale Galliera di Genova, inaugurato come opera pia tra il 1877 e il 1888, che da allora svolge all'interno della città un importante ruolo assistenziale. L'edificio presenta un interessante apparato artistico realizzato da importanti scultori e pittori dell'epoca, tra cui Giuseppe Isola, Lorenzo Garaventa, Giovanni Cevasco, Giulio Monteverde, Antoine Horace Vernet.

Cevasco realizza per volere della marchesa Brignole-Sale il gruppo scultoreo della Pietà, posto all'interno della cappella dell'ospedale, il quale rispecchia le tendenze artistiche della metà dell'Ottocento. La fede, la speranza e la carità sono tematiche cardine dell'attività politica ed artistica dello scultore genovese: non solo è presidente degli asili infantili di Genova e sostiene attivamente gli enti di carità territoriali, ma ebbe anche un rapporto profondo con le istituzioni ecclesiastiche, arrivando ad avere uno scambio epistolare con l'allora pontefice Pio IX, definito il papa liberale e riformista ${ }^{11}$, come dimostrato da un ritrovamento autografo in cui il Papa scrive una lettera di risposta allo scultore in merito all'elezione di Angelo Brofferio, deputato italiano anticlericale, che sia Pio IX sia Cevasco disapprovano. 
西 diverse visioni che caratterizzeranno il Risorgimento e il processo che porterà all'Unità d'Italia, il continuo confronto tra ideali repubblicani e monarchici, clericali e anticlericali. La partecipazione attiva del Cevasco alla politica genovese ben evidenzia come gli artisti dell'epoca vivessero con genuino interesse l'evolversi di un sentimento unitario che, a partire dai moti del 1820-1821 e attraverso quelli del 1830 e 1848, porteranno ad un crescente senso di appartenenza nazionale e infine, nel 1861, alla tanto agognata Unità d'Italia ${ }^{12}$.

\section{Il carteggio politico e istituzionale di un assessore-scultore}

Cevasco fu consigliere nella giunta comunale genovese, dal 1848 al 1878. La sua parabola politica è parallela a quella artistica, egli intrattiene rapporti epistolari con personaggi illustri genovesi e italiani, tra i quali Garibaldi, Nino Bixio, Vincenzo Ricci, Andrea Charvaz e Cavour. Nella carriera politica dello scultore è infatti molto importante l'interesse per tematiche sociali e pedagogiche. Inoltre, si menziona che Cevasco avesse un forte interesse conservativo per i beni culturali, tanto è vero che emana all'incirca nel 1850/1851 un provvedimento al fine di conservare, catalogare e adibire una sala apposita per contenere le opere d'arte sparse nel municipio di Genova. Come emerge in una sua lettera del 22 marzo 1851 alla giunta comunale:

Bisogna raccogliere nell'atrio di codesto Palazzo Municipale tutti quegli oggetti appartenenti al comune che possano interessare le arti belle e la pratica storica, sarebbe gentile e lodevole pensiero. Non pochi di questi oggetti preziosi sono sparsi in diversi locali; quasi dimenticati con grande desiderio della nostra città. Nel Portico del palazzo Comunale esiste per esempio il busto marmoreo del tanto benemerito cittadino Ettore Vernazza, collocato indecentemente sopra di un orinatoio, $[. . .]^{13}$.

La lettera prosegue auspicando una collocazione dignitosa del busto di un personaggio che fu tra i maggiori benefattori della città.

Il consiglio comunale inizialmente è incerto, ma poi decide di stanziare $\mathrm{i}$ fondi necessari all'impresa e le opere in questione sono: il pallio donato all'imperator Paleologo dalla Repubblica di Genova, una tavola bronzea illustrata dall'artista Giuseppe Banchero, il busto di Cristoforo Colombo e il codice Colombo, il sacro catino che si pensava fosse di smeraldo, una croce bizantina, lapidi inviate al comune dal generale Alfonso La Marmora, un San Benedetto e un San Francesco di Luca d'Olanda, un trittico d'Alberto Dürer di argomento sacro e altre opere, tra cui una tavola di Bernardo da Papia rappresentante la vergine e i santi ${ }^{14}$. La sala in questione denominata dal Cevasco Sala Colombo o Museo Colombo ${ }^{15}$.

Nel 1853 il consenso di Cavour è ai minimi storici, sia per la pressione tributaria applicata dal Ministro sia per la crisi agraria che colpisce il Piemonte a causa di un parassita, l'oidio che distrugge i vigneti e i raccolti. Questo provoca un drastico innalzamento dei prezzi del pane, del vino e del frumento, così la rabbia e il malcontento si riversano direttamente sul Primo Ministro ${ }^{16}$. In questa situazione anche a Genova cresce il malcontento verso il Ministro, la stampa, prevalentemente di sinistra, si scaglia contro di lui, accusandolo di essere la causa principale di questa 
situazione. La condizione si aggrava quando il Ministro propone di spostare l'Arsenale militare da Genova a La Spezia. L'otto dicembre del 1854 si svolgono le elezioni per la quinta legislatura, a Genova l'astensionismo è molto alto, in tutti i collegi si eleggono candidati di schieramenti antiministeriali. Alcuni consiglieri comunali tra i quali Casaretto e presumibilmente Cevasco sono candidati dello schieramento di Cavour e optano per elezioni suppletive che si tengono alla fine del 1854, ma viene comunque eletto un candidato dello schieramento opposto. In una lettera del 3 luglio 1854 Cavour si rammarica così con Cevasco:

Torino, 3 luglio 1854

Ill.mo Signore,

I lavori e le preoccupazioni di cui altrimenti dicevamo, non mi lasciano tempo per rispondere prima d'ora alla sua confidenziale e riverente. Temo perciò che la risposta che io sto per fare alla vostra nuovissima interpellanza giunga troppo tardi. Comunque sia non voglio tralasciare di manifestarle quanto io valuti il sentimento che la muove a volere promuovere a Genova un pubblico degno di simpatia e di affetto per la casa regnante e infine per la nostra Augusta regina. Temo però che ella non possa riuscire nel suo intento, stante i molti malumori che fermentano nel corpo municipale di Genova: che anzi dubito che ciò stante non sii prudente mettere avanti una proposta che produrrebbe mediocre effetto, quando non fosse accettata senza discussione. Ciò detto mi affido interamente ed alla sua prudenza ed alla sua devozione per quelle cose a cui il nostro paese va debitore, da essere invidiato fra tutti quelli d'Europa. In fretta le invio i sensi della mia eterna stima.

C. Cavour ${ }^{17}$.

A Genova in Consiglio Comunale dopo le elezioni supplettive del 1854, viene eletto Angelo Brofferio, nato a Castelnuovo nel 1802, poeta e giornalista che aveva fondato il giornale a sostegno dell'indipendenza italiana Il Messaggiere Torinese. Egli è apertamente contro la politica di Cavour, si professa progressista e liberale ${ }^{18}$ e anticlericale anche se in questa fase cresce all'interno del ceto moderato il consenso per Pio IX. In una lettera del nove maggio del 1856 il papa scrive a Cevasco esprimendogli la sua contrarietà sull'elezione di Brofferio:

Amico carissimo,

mille grazie con tutto il termine del sentimento ammirevole che traspira dalle sue parole, mi compete abbastanza se intervenire che non mi do pace di pensare alla vile nota del Brofferio, disprezzata già da quattro anni. Uomo senza carattere e senza pregi e giunto alla stregua di una carriera immeritata. La chiesa si oppone alle istanze proposte per la città di Genova e Savona, sarebbe importante agire per la salute della città di Genova. Felicità di cuore per i momenti belli e per un'avvenire luminoso. Cordialissimi saluti, Il Pontefice. A Genova e alla Gazzetta ${ }^{19}$.

La sua corrispondenza, sia di natura politica che artistica, è molto vasta. I rapporti con Vincenzo Ricci, nobile genovese e figura di spicco tra i democratici liguri e deputato ministro e poi deputato all'opposizione di sinistra ${ }^{20}$, sono molto frequenti, come emerge anche dalla sua corrispondenza, per esempio in una lettera indirizzata al nobile del 22 gennaio 1855:

Illustre Signor Vincenzo cortesissimo,

Io non ho parole per esprimerle la mia riconoscenza per il disturbo che vi siete dato a mio riguardo, e per le parole incoraggianti che la sua bontà mi rivolge e spesso mi sono di lieve conforto perché mi vengono da un personaggio per il quale nutrii sempre stima e rispetto, per la generosità del suo cuore e per la schiettezza del suo carattere. Io spero pertanto Signore, che ben nota cortesia mi avrà scalfito l'audacia se osai rivolgermi a lei e disturbarla per un oggetto mio particolare e se per giunta le è apparso così poco modesto; ma lei ben sa che l'artista ferito nell'amor proprio si allontana talvolta [...] Vorrà venire in soccorso della Liguria vostra la quale 
benedirà la mano che l'avrà beneficiata. Farà una pia elemosina fatta bene. Perdoni il disturbo e nel pregarla di volermi usare il favore di presentare i miei rispetti all'ottimo fratello, signor Giovanni ${ }^{21}$.

Probabilmente Cevasco in questo frangente ha donato un'opera d'arte al Ricci, il quale forse ha fatto una critica ad una sua scultura. Il rapporto che intercorre tra i due si suppone fosse collaborativo e di amicizia. Non è insolito che nella sua carriera lo scultore donasse alle personalità le sue opere.

Giovan Battista Cevasco è altresì un attivo sostenitore dei moti risorgimentali e intrattiene nutriti scambi epistolari con Garibaldi e Nino Bixio, con cui sembra essere in confidenza. Queste lettere sono interessanti non solo per capire meglio come egli agisca politicamente in qualità di consigliere municipale, ma anche per inquadrare le vicende storiche genovesi relative a questo periodo. Lo scambio epistolare con Giuseppe Garibaldi è composto da diverse lettere che egli ha inviato al Cevasco, ma purtroppo non sempre sono presenti le risposte.

Le lettere che seguono sono relative alla fase antecedente la spedizione dei Mille:

Nizza, 6 dicembre 1854

Carissimo Cevasco,

ebbi il gentilissimo vostro foglio ed i numeri della stampa ove avete degnato onorare il mio nome con cenni sul poco fatto da me in America. Io ve ne sono grato davvero e con più comodo io vi paleserò alcune inesattezze inserite in quella narrazione. Circa ad informarvi sugli indirizzi dei Pittaluga e il Sig. Borbotta, compiacetevi di cercare del D. Cenni, aiutante mio di campo, domiciliato in Genova. Accetto coll'anima l'augurio vostro sulla prossima nostra pugna e sono Vostro.

G. Garibaldi ${ }^{22}$.

2 In una lettera da Nizza del 24 agosto 1856 Giuseppe Garibaldi si scusa con Cevasco per la risposta tardiva e aggiunge che, appena di ritorno a Genova, chiederà di vedere il busto fatto dallo scultore per Varella, suo amico.

Nizza, 24 agosto 1856

Caro Cevasco,

È meglio tardi che mai, ed io le avrei risposto prima, se non fossi stato spinto in un turbine d'affari che mi hanno levato da ogni dovere. Io vi ringrazio tanto per la vostra amicizia e disponibilità. Io vi ringrazio tanto per la stratta di mano del nostro amico e per la vostra. Spero tra giorni di essere in Genova ed avrò il piacere di vedere il busto di Varella, amico mio, e darvi personalmente i miei ringraziamenti ${ }^{23}$.

Anche Nino Bixio ha rapporti epistolari con il Cevasco, come si vede in alcune lettere, in particolare una da Torino del 19 novembre 1864, nella quale, il grande garibaldino, agitatore un tempo di vivaci fermenti rivoluzionari, ormai eletto deputato per il secondo collegio di Genova, e quindi uomo d'ordine, si propone come mediatore per un busto eseguito da Cevasco per Vittorio Emanuele II.

Torino, 19 novembre 1864

Caro Cevasco,

Mille grazie e mille scuse del mio ritardo a rispondere: accetto con piacere di presentare in tuo nome al Re la fotografia del busto effigiato da te. Mandamelo quando credi e io troverò il modo di presentarlo. Mi gode l'anima che l'ignaro Longoni sia favorevole alla convenzione ed al trasferimento. Segno che comprende l'Italia! Se hai occasione salutamelo affettuosamente. Tu sai che il Longoni è il mio primo ed antico capo $^{24}$.

Giovanni Battista Cevasco è in contatto anche con Raffaele Rubattino, importante armatore genovese, uomo del movimento mazziniano e repubblicano che sostenne finanziariamente con le sue stesse navi l'impresa dei Mille. 

lo scultore gli spedisce a casa un tronco di colonna marmoreo probabilmente con un'effigie e per sostegno di un busto:

Carissimo Raffaele,

Io mi sono già congratulato per voi mediante una corta visita che avete ricevuto dalla splendida dimostrazione, per vero meritatissima, datavi spontaneamente da Vittorio Emanuele e vi assicuro che ne ho provato vero piacere. Mi fece rabbia, lo confesso l'indifferenza dei giornali locali che non si degnarono di accompagnare con qualche riga di approvazione la notizia dell'atto sovrano, che senza un ottimo cittadino a parte la modestia cotanto benemerito. In altri paesi si opera trionfalmente, tale indifferenza mi ha spinto a negare alcune parole, che attinsi nel fondo del cuore e ad inviarle al Cavalier Gandolfi [...]

Io vi prego di gradire questo tenue ma schietto attestato di stima e di amicizia quando anche non ammirasse colla vostra modestia, la quale del resto non potrebbe trovare in quei pochi periodi nulla ma proprio nulla d'adulatorio, sebbene l'immagine della verità più pura. [...] Anche l'ottimo Sig. Giacomo Baratta ha provato un vivo piacere all'annuncio della vostra onoreficenza e mandò a casa vostra la sua cartella di visita. Il tronco di colonna per voi lo feci riportare a casa vostra, non vidi ancora l'avvocato Cossuti perché assente. [...] State bene e tenetemi sempre aggiornato.

Affezionatissimo

Cevasco $^{25}$.

Cevasco è in contatto con personaggi di diverse cittadine, tra i quali Giuseppe Airenti, avvocato e sindaco di Porto Maurizio ${ }^{26}$. L'otto maggio del 1866, Cevasco gli invia una lettera, chiedendo aiuto per le famiglie dei soldati impiegati nella terza guerra d'indipendenza contro l'Impero austriaco dal 20 giugno al 12 agosto $1866^{27}$ :

Ill.mo Signor,

quando io per preghiera dell'illustrissimo signor sindaco di questa città, di buon grado ho accettato di iniziare la costituzione di un comitato, il quale si adoperi a procurare soccorso alle famiglie dei poveri soldati che vanno straordinariamente chiamando sotto le armi; mi pregio invitare la Signoria Vostra Illustrissima, a voler accettare di fare parte del predetto comitato. Confido che la illuminata sua carità non vorrà negare il suo concorso ad un'opera tanto necessaria e benefica [...].

Di Vostra Signoria Illustrissima

Cevasco G. B.

Queste lettere dimostrano quanto il Cevasco fosse in realtà una personalità influente a Genova, sia dal punto di vista politico, sia dal punto di vista artistico.

La sua rete di contatti molto ampia lo rivela un liberale moderato, nonostante i suoi precedenti di acceso sostenitore del pensiero repubblicano e mazziniano. (Francesca Gastaldo)

\section{La musica, il patriottismo e Mazzini}

Nonostante leciti scetticismi, è un dato di fatto che le rivoluzioni per essere tali hanno bisogno del contributo del popolo.

Se pensiamo all'Italia degli anni precedenti al 1861, dobbiamo probabilmente immaginarci una nazione «senza nazione», un insieme di tradizioni, mentalità comuni e una storia condivisa. Tutti gli italiani vivevano in una nazione che si faceva portatrice di tutti i fasti del suo passato, esponendoli senza veli nelle vie e piazze delle sue città e paesi; una cultura secolare che si manifestava nelle forme dell'architettura che 
scandiva la vita quotidiana di tutti già a partire dall'epoca romana, passando per il Medioevo e il Rinascimento, senza dimenticare le grandi opere urbanistiche del Seicento e del Settecento. Questo è ciò che vediamo con grande chiarezza oggi, ma non doveva apparire altrettanto consolidato e facilmente identificabile da parte di quel popolo che avrebbe di lì a poco dato l'unità alla propria nazione; dobbiamo, invece, pensare che più probabilmente tutte queste nobili vestigia di cui l'Italia era portatrice erano presenti in modo più o meno ignorato da parte del popolo.

51 Negli anni a cavallo fra il XVIII e il XIX secolo, vi è molta confusione nell'uso del termine «nazione» in riferimento all'Italia, si parla anche di nazione piemontese o romana, confusione che viene chiarendosi agli albori dell'ottocento grazie al nuovo linguaggio politico e ai suoi promotori, che puntano all'Italia come nazione, appunto, promuovendo la comunità di riferimento, «per questo la loro identità socioprofessionale primaria è legata al sentirsi parte di una koinè culturale che comunica attraverso il ricorso alla lingua italiana, nobilitata dal fatto che sin dal XIV secolo essa può fregiarsi di capolavori letterari di prim'ordine ${ }^{28}$. Fanno caso a sé la lingua e la letteratura, che provano l'esistenza della nazione italiana e ne motivano il riscatto politico. Tuttavia il periodo postunitario vede una percentuale bassissima di popolazione che utilizza la lingua italiana, tra il 2,5\% e il 9,5\%, tutti gli altri utilizzano ancora $\mathrm{i}$ dialetti e le lingue straniere. I patrioti parlano a masse che a stento comprendono l'italiano, su queste fragili premesse però il Risorgimento compie una missione «impossibile» nell'arco di meno di cinquant'anni (1815-1861).

Cosa può, allora, aver costruito un sentimento nazionale tanto potente da portare a un movimento di liberazione nei confronti dell'invasore straniero?

Ciò che possiamo affermare, certo semplificando, è che a fronte di un singolo moto ribelle si possono identificare diverse componenti: quella economica che ha ricadute sulle condizioni quotidiane di vita, quella ideologica che fonda tutta la sua esistenza sull'aspetto culturale, e quella sentimentale, la meno inquadrabile ma forse la più indispensabile, e quella più rilevante nello studio dei sentimenti patriottici che hanno trovato sfogo nelle arti e nella letteratura. I portavoce intellettuali e politici del nazional-patriottismo hanno saputo, infatti, presentare gli ideali unitari facendo appello «all'universo pre-razionale delle emozioni»» Se, dunque, è vero che le rivoluzioni devono essere fatte dal popolo per il popolo, questo deve essere non solo coinvolto, ma anche culturalmente formato, attraverso strumenti come l'arte, il teatro e la musica.

Il lessico politico vede un mutamento irreversibile, che trascina con sé anche un altro termine, quello di «patria», utilizzato in epoca medievale con accezione politica, in quanto era già consolidato il concetto di «morire per la patria» e dopo la Rivoluzione Francese i termini nazione e patria si congiungono ed entrano in stretto rapporto, al punto che si parla di pensiero nazional-patriottico.

Prende forma quella che George L. Mosse definisce «estetica della politica» ${ }^{30}$, che muove anche dal Romanticismo europeo, in particolare nella concezione di un'arte popolare, per il popolo, che raggiunga, cioè, la più grande popolazione possibile. Bisogna tenere a mente che l'artista della Restaurazione non è più un "mantenuto» dalla nobiltà in cambio delle sue creazioni, ma un «libero professionista» che deve trovare il lavoro da sé, e per far questo deve anche allargare il suo pubblico. Ciò avviene adattando il tipo di narrazione, più legata alle tradizioni nazionali e al discorso patriottico, in quanto argomento di attualità che accende gli animi del popolo-pubblico, 
e in cui inoltre molti artisti sono intimamente coinvolti, tanto da rischiare prigionia, esilio e morte. Sono le opere che scaturiscono da questo ambiente, poesie, romanzi, melodrammi, dipinti e sculture, che costituiscono l'estetica risorgimentale, a opera di artisti come Ugo Foscolo, Alessandro Manzoni, Francesco Hayez, Giuseppe Verdi e molti altri. La diffusione di queste opere è interessante poiché avviene grazie alla propaganda derivante dalle organizzazioni mazziniane, in primis la Giovine Italia in città portuali e universitarie come Genova, Livorno e Pisa; ma anche attraverso la messa in scena dei melodrammi musicali, che hanno in Italia un particolare successo dovuto anche alla costruzione di ben 613 teatri tra il 1816 e il 1868, luoghi di cultura non esclusivi dati i prezzi dei biglietti molto vari e l'accessibilità da parte di analfabeti per la natura delle opere rappresentate. Vanno inoltre ricordati i predicatori itineranti e un'altra serie di media «secondari» come le stampe monocromatiche e gli artisti girovaghi di strada che portavano in scena nelle piazze italiane spettacoli che riprendevano per lo più musiche di successo sempre provenienti da opere teatrali. Questa diffusione a mezzo artistico definisce una geografia dell'estetica patriottica italiana che però esclude le campagne, anzi resta per lo più urbana. Sempre secondo Banti, laddove giunge il discorso nazionale è capace di animare gli uditori perché fa leva su miti e simboli potenti: la nazione come comunità di discendenza, aprendo ad un nesso biologico che prende poi forma in termini come madre-patria, padri della patria, fratelli d'Italia. Alcuni dei più chiari esempi di retorica nazionale vengono da Mazzini in brani come Agli italiani, $e$ specialmente agli operai italiani:

Dio v'ha data, come casa del vostro lavoro, una bella Patria, provveduta abbondantemente di tutte risorse, collocata in modo da esercitare influenza su tutte le terre abitate da uomini come voi, protetta dal mare e dall'Alpi, confini sublimi che la dichiarano destinata ed essere indipendente: questa vostra Patria fu grande e libera un tempo; grande e libera quando le nazioni, ch'ora vi stanno innanzi in tutto, erano piccole e serve; e voi non la curate, non l'amate, non la conoscete, non ne sapete la storia, e lasciate ch'essa si stia decaduta, avvilita sprezzata, malmenata da principi e governi imbecilli, tiranneggiata e spolpata d'oro e di sangue da quanti stranieri hanno avidità d'occuparla e di dominarla. Dio v'ha fatti ventidue milioni d'uomini, con una stessa fisionomia per conoscervi, con una stessa lingua madre di tutti i vostri dialetti per intendervi, con una stessa indole svegliata, attiva, robusta, per associarvi e lavorare fraternamente al vostro miglioramento in Unità di Nazione; e voi vi state divisi, separati da leggi, da dogane, da barriere, da soldatesche, mal noti gli uni agli altri, anzi spesso ostili tra voi, ubbidienti a vecchie e stolte rivalità fomentate, perché siate sempre deboli, dai vostri padroni, e vi dite romagnoli, genovesi, piemontesi, napoletani, quando non dovreste dirvi ed essere che ITALIANI ${ }^{31}$.

57 Un'opera centrale nel pensiero mazziniano a proposito della musica è Filosofia della musica del 1836, dall'analisi della quale emergono alcuni aspetti rilevanti nella concezione dell'espressione artistica musicale di Mazzini, che avrà poi una notevole ricaduta sulla composizione di musica e testi soprattutto degli inni risorgimentali.

Mazzini pubblica questo saggio sulla rivista «L'Italiano» di Michele Accursi che la fonda a Parigi, una delle riviste che compaiono, e spesso compaiono in un arco temporale molto breve, in quegli anni subito seguenti la rivoluzione del 1830, come ricorda Claudio Strinati:

[...] in continuazione compaiono e scompaiono carbonari, fuoriusciti, liberi pensatori, rivoluzionari a tempo pieno, massoni non certo estranei alle attività mazziniane, cospiratori perennemente tenuti sotto perenne osservazione dalle autorità di polizia, intellettuali e filosofi animati da aspirazioni universalistiche e 
palingenetiche. Ricorrono sovente a piccoli fogli stampati da audaci e infaticabili tipografi, che durano magari lo spazio di un mattino, per veicolare idee e progetti nella pressoché assoluta certezza che le pubblicazioni non potranno durare più che tanto, o perché non sostenute economicamente o perché soppresse da un potere politico dittatoriale e pervasivo ${ }^{32}$.

Il XIX secolo è, infatti, anche il secolo del successo dell'editoria musicale, vero e proprio terreno su cui viaggia la critica e il pensiero in materia musicale, nel 1823 esce la bolognese Polinnia Europea ossia Biblioteca universale di musica storico scientifico letteraria e curioso dilettevole, dal carattere scientifico-letterario rigoroso, sancendo la nascita anche delle prime riviste ad argomento musicologico.

Nei primi vent'anni dell'Ottocento nascono i giornali teatrali che introducono anche la recensione delle rappresentazioni teatrali e sinfoniche, determinando la nascita di critici musicali, come Carlo Collodi, che dirige a Firenze «Lo Scaramuccia» tra il 1853 e il 1859, all'epoca noto esempio di periodico musicale. Insieme ad altri periodici inglesi come «The Tatler» (1709-1711) di Richard Steele e «The Spectator» (1711-1714) di Joseph Addison, anche le riviste musicali italiane si allontanano dal linguaggio accademico e si aprono al pubblico borghese e non di settore. Nascono così «Il Censore Universale dei Teatri» (1829-1838), che diventerà poi il "Corriere dei teatri» (1839-1840), volto a fornire informazioni di vario genere sulle produzioni teatrali in corso, dai cantanti agli impresari; e «Il Barbiere di Siviglia» (1832-1834) che diventa dopo soli due anni «Figaro», composto da quattro pagine nelle quali hanno spazio un editoriale affidato a firme illustri e notizie sui teatri locali. Negli anni ' 40 prendono sempre più spazio nelle riviste musicali la critica e la cronaca delle rappresentazioni teatrali e dei concerti, ne sono chiari esempi la «Gazzetta musicale di Milano» fondata da Tito Ricordi nel $1842 \mathrm{e}$ il «Boccherini», organo di stampa ufficiale della Società del Quartetto di Firenze, attivo dal 1862 al 1882, sulle cui pagine compaiono oltre che articoli sulle opere contemporanee anche riflessioni critiche sulle opere del passato.

61 Accursi è all'epoca un mazziniano tra luci e ombre. Inizialmente affiliato alla Carboneria, una vera e propria setta abituata ad agire "nell'ombra», in netta opposizione al sentimento mazziniano dell'agire uscendo allo scoperto, era stato arrestato e imprigionato più volte, fino all'ultima in occasione della fallita rivoluzione del 1831. Venne poi liberato da Mons. Cappelletti e inviato prima in Svizzera e poi a Parigi, dove è probabile che avesse il compito di sorvegliare proprio Mazzini. Il periodo parigino fu il preambolo di una peregrinazione del patriota genovese, cacciato dalla capitale francese, forse proprio a causa dello spionaggio ad opera di Accursi o di altri, andò in Svizzera e da lì a poco dovette andarsene e approdò a Londra. Filosofia della musica viene scritto prima di questi avvenimenti e del perenne esilio di Mazzini, prima di quella cocente delusione che ne deriverà e che gli arrecherà un tracollo fisico $\mathrm{e}$ morale, ed è infatti uno scritto da cui emerge solo una grande carica di entusiasmo, fiducia e speranza. «L'Italiano» incontra Mazzini in questo contesto, che lo dota inoltre di un inserto dal titolo Il Precursore, "giornale della rigenerazione italiana». Va ricordato che il riferimento primario in queste riviste è l'Antologia, nata a Londra nel 1821 grazie al marchese Gino Capponi e Giampiero Viesseux, e con la collaborazione di una personalità del calibro di Ugo Foscolo.

Nella pubblicazione di Mazzini si possono trovare alcuni punti di riflessione sul pensiero patriottico associato alla musica, il primo dei quali è il concetto di «rigenerazione italiana»: 
La musica per Mazzini ha una funzione fondamentale e indispensabile nella formazione culturale e ideale del cittadino da lui vagheggiato e ancora lontanissimo dall'assumere una fisionomia attendibile e plausibile e soprattutto coerente con gli ideali propugnati dal grande patriota genovese ancora agli inizi della sua sbalorditiva carriera di agitatore di libertà e progresso ${ }^{33}$.

Il concetto di rigenerazione ha anche una sua particolare chiave di lettura, legata al senso di religiosità e in particolare all'accostamento ideologico al cristianesimo, che passa per il concetto chiave di 'martirio', in questo caso dei patrioti che hanno testimoniato la loro fede politica con la loro stessa morte, trasformando in qualche modo la rinascita della nazione in una vera e propria resurrezione, nel quale significato va visto anche l'uso del termine Risorgimento stesso. Inoltre

[...] affinché l'azione degli eroi che si sacrificano per la patria abbia un senso, è necessario che tale azione sia ricordata, commemorata e costantemente portata ad esempio. È necessario, quindi che si costruiscano riti della memoria, monumenti commemorativi, testi che traccino una mappa delle morti degne di essere ricordate ${ }^{34}$.

Riferimenti alla sacralità dell'arte musicale ricorrono lungo lo scritto mazziniano, ed hanno il loro apice nel passaggio seguente:

L'arte che trattate è santa, e voi dovete essere santi com'essa, se volete esserne sacerdoti. L'arte che v'è affidata è strettamente connessa col moto della civiltà, e può esserne l'alito l'anima il profumo sacro, se traete le ispirazioni dalle vicende della civiltà progressiva, non da canoni arbitrari, stranieri alla legge che regola tutte le cose. La musica è un'armonia del creato, un'eco del mondo invisibile, una nota dell'accordo divino che l'intero universo è chiamato ad esprimere un giorno; e voi, come volete afferrarla, se non innalzandovi alla contemplazione di questo universo, affacciandovi colla fede alle cose invisibili, abbracciando del vostro studio, dell'anima vostra e del vostro amore tutto quanto il creato ${ }^{35}$ !

Altre influenze nel Mazzini che scrive questo saggio sulla musica, sono il pensiero di Vittorio Alfieri nel Misogallo ed Hector Berlioz. Dal primo prende senz'altro il sentimento di superiorità italiana sui francesi "prepotenti, falsi, arroganti e nemici» ${ }^{36}$; dal secondo invece prende il passaggio dal concetto di idea a quello di ideale che pervade la partitura della Sinfonia fantastica. Si legge infatti nelle prime pagine mazziniane che «[...] l'arte sovrana, Byroniana, profonda, l'arte che solca e scava, l'arte di insistere sul concetto, con incremento progressivo di forza, finché s'addentri, si incarni, s'invisceri in voi, è negletta e perduta» ${ }^{37}$. Si apre così la strada a uno dei concetti fondamentali di cui è o vuole essere interprete la musica patriottica del periodo risorgimentale, secondo cui non vi deve essere inutile semplificazione o banalità nella composizione di un'arte popolare, ma una forte ispirazione e una serietà di contenuti. Mazzini fa perno proprio su Byron, cui si legò anche Berlioz nell'opera Child Harold per la composizione della sinfonia Harold en Italie, facendone un esempio di pensiero ed azione, arrivando qualche decennio dopo a darne il nome alla rivista che fonderà a Londra nel 1858.

Nonostante una passione sincera per la musica tutta, vissuta da Mazzini anche nella sua dimensione intima e riservata, come ci ricorda la sua passione per la chitarra, strumento emblematico della musica da camera e salottiera, la Musica con la $\mathrm{M}$ maiuscola di cui Mazzini si occupa nel suo saggio è la musica pubblica, con la quale il suo amore per essa va a compimento in uno spirito di condivisione con le masse, finalmente risvegliate dalla volontà di conseguire l'unità repubblicana della nazione. In questo testo traspare un trasporto e una passione travolgente del patriota che ripone una fiducia totale nell'espressione musicale come elemento comune e unificante di 
questo processo storico esaltante nel quale Mazzini crede fermamente, soprattutto nel 1830, quando scrive: «il supremo insegnamento mazziniano, infatti, è l'anelito alla libertà. Ed egli ritiene che tale anelito possa essere meglio instillato in chi abbia assimilato una sorta di senso del dovere estetico» ${ }^{38}$.

La musica di cui parla Mazzini è l'opera lirica, quell'opera italiana che dopo aver influenzato la nascita della grande scuola operistica austriaca, ha una grande influenza e un altrettanto grande successo in Francia, al cui apice intellettuale mette la figura di Rossini, visto come un eroe capace di superare le decadenze fatte di abusi di tecnicismi e inutili quantità di note, contro cui Mazzini si schiera ripetutamente nel corso del suo scritto. Rossini riporta la bellezza che si confà ad un'arte che protende al divino come deve essere la musica, liberandola dalla superficialità cui l'aveva condannata la scuola metastasiana italiana. Proprio Rossini, che fu sempre un conservatore sia artisticamente che politicamente, ripensò l'opera come un'espressione del tutto progressista.

Gli autori che Mazzini identifica come primi eredi di queste doti rossiniane sono quelli che stanno in quegli anni trionfando a Parigi, Luigi Cherubini, Gaspare Spontini, Giovanni Paisiello, Niccolò Piccinni e Antonio Sacchini, fino ad arrivare alla grandiosa sintesi di Bellini e Donizetti. Nel 1835 a Parigi si susseguono I Puritani di Bellini e dopo due mesi Marin Faliero di Donizetti, suscitando un grande successo di pubblico.

Nel rapporto fra musica e popolo Mazzini coglie un aspetto fondamentale: la musica che egli disprezza, per tutti i difetti già elencati, ha una valenza politica forte perché gli permette di seguire i gusti e i desideri del popolo e di tutti gli ascoltatori. Per questo aspetto, seppur deplorevole ai suoi occhi, l'opera lirica emerge perciò come l'unico ambito musicale adatto a interpretare il nuovo necessario impegno civile popolare. Mazzini in questo si dimostra un attento uomo politico e intellettuale, capace di prevedere il necessario passo successivo che in effetti la musica compirà da lì a breve. Verdi è in quegli anni ancora solo un capacissimo docente e Wagner si è appena approcciato alla composizione musicale, ma saranno loro le nuove fulgide stelle che porteranno a compimento quell'unità artistica e civile che il patriota genovese auspica e vede come unica via percorribile per la musica in queste pagine parigine.

Lo scritto di Mazzini porta, a ragion, veduta, nel titolo la parola «Filosofia» poiché parte da uno degli interrogativi più classici in filosofia estetica: può esistere il bello universale, qualcosa che piaccia veramente a tutti? Ovvero «se la bellezza, e di conseguenza il criterio della validità estetica, sia da considerarsi nell'ambito del concetto o in quello dell'opinione» ${ }^{39}$.

La Filosofia della Musica in parte insegue una sorta di lucido e intelligente delirio visionario dove genialità e banalità si sovrappongono e si scontrano pericolosamente. Dall'altro tiene i piedi ben saldi per terra e descrive una realtà di fatto di cui intuisce lucidamente gli sviluppi.

Verdi e Wagner, ciascuno nel proprio ambito di competenza tecnica, di eletta ispirazione e di consapevolezza teoretica, realizzeranno veramente le istanze poste da Mazzini, culmine di un Romanticismo che Mazzini stesso in realtà disprezzava o quanto meno svalutava ${ }^{40}$.

Già nelle prime pagine, nell'ideologia mazziniana, fa anche la sua comparsa un pensiero anti-romantico:

Quando il romanticismo gittò sulla mensa dei letterati il pomo della discordia, i letterati erano Greci o Romani bastardi, non Italiani, non Europei del secolo XIX. L'antico era despota. - L'elemento del mondo moderno cancellato. L'Arte cristiana, 
l'Arte libera, l'Arte umana affogava sotto i rottami del mondo Pagano. Il romanticismo, come gli invasori settentrionali sul finir dell'impero, venne a por mano in quelle morte reliquie e le scompigliò; dissotterrando l'individualità conculcata, e mormorando all'intelletto, applicata all'Arte, una parola obliata quasi da cinque secoli, lo riconsecrò e gli disse: va oltre: l'universo è tuo: non altro. E allora gli ingegni divagarono per quante vie s'affacciavano: salirono al cielo, e si ravvolsero nelle nuvole del misticismo; scesero, rovinando all'inferno, e ne trassero il ghigno satanico e quello sconforto senza fine che domina in Francia tanta parte di letteratura; si prostrarono alle reliquie dell'evo medio, chiesero l'ispirazione ai rottami dei chiostri e dei monasteri. Da tutti questi tentativi, come che incerti, o esclusivi, e talora retrogradi, esciva, presagio dei lavori futuri, e indizio di una coscienza e di una potenza rinata, un pensiero: l'io restituito alla propria missione. A quanti interrogavano: in chi avete fede? Gli ingegni potevano almeno rispondere la risposta del barbaro: in noi. - Bensì quando s'avvidero che il vuoto durava, che essi non sapevano colmarlo, e che i desiderii della crescente generazione non s'appagavano di quei tentativi, ristettero sfiduciati e ristanno ${ }^{41}$.

Nove anni dopo, in occasione della sua cronaca delle nozze tra Vittorio Emanuele e Maria Adelaide, Federigo Alizeri usa queste parole per descrivere la lingua italiana:

E siccome quel ch'è di forma e di locuzione nostra, e la nettezza, e 'l dolce impasto della nostra favella tanto s'afforza co' libri dell'antica Roma quanto traligna per imitazione de' barbari, così mi piacquero i latini componimenti alternati agli italiani per significare che una è l'indole, uno il prezzo delle due lingue ${ }^{42}$.

Questi concetti danno un'ulteriore prova di quanto il pensiero mazziniano espresso nel volume Filosofia della musica, non fosse affatto sconosciuto agli intellettuali del tempo, che anzi lo abbracciano come negli esempi qui citati. Il carisma di Mazzini è conosciuto oltre i confini, come non è sconosciuto il suo amore per la musica, che indubbiamente ha contribuito a diffondere il suo pensiero fra quanti già lo seguivano affascinati, anche in fatto di critica musicale. Auspicando inoltre l'unità delle arti, questo pensiero va a estendersi oltre la musica, e tocca la critica artistica, come sembra dimostrato dalle affermazioni di Federigo Alizeri, uno degli eruditi di arti figurative del risorgimento genovese. (Chiara Capobianco)

\section{NOTE}

1. L. T. Belgrano, Della vita e delle opere del Marchese Gerolamo Serra, Genova, Sordomuti, 1859, pp. 44-46.

2. Della vita di Giuseppe Mazzini per J. W. Mario, Opera illustrata con ritratti e composizioni d'insigni artisti, Milano, Sonzogno, 1886.

3. R. Bottini, Emanuele Celesia patriota, letterato, educatore e filantropo, Genova, Edizioni De Ferrari, 2019.

4. M. Dalai Emiliani (a cura di), Federigo Alizeri (Genova 1817-1882) un «conoscitore» in Liguria tra ricerca erudita, promozione artistica e istituzioni civiche, Genova, Istituto di Storia dell'Arte dell'Università di Genova, 1988.

5. A. Grimaldi, Michele Giuseppe Canale, La vita, le battaglie e gli studi di un Genovese nell'Ottocento, Milano, Biblon, 2016. 
6. E. Celesia, Diario degli avvenimenti di Genova nell'anno 1848, in Comune di Genova (a cura di), Genova nel 1848-1849, Torino, 1950, pp. 12-54.

7. F. Alizeri, Commentario delle cose accadute in Genova in marzo e aprile del 1849, scritto per ordine del Municipio (con introduzione e appendice), in Genova nel 1848-1849, cit., pp. 69-332.

8. L'Album di Elena d'Aste, manoscritto, Civica Biblioteca Berio, Sezione Conservazione, m.r, cf. bis. 3.1. è un quaderno femminile, dove scrissero di volta in volta i loro pensieri, le loro frasi e le loro liriche gli amici del prof. Ippolito d'Aste, padre di Elena, professore di calligrafia e drammaturgo, amico di Gian Carlo Di Negro, fondatore, insieme a Federico Alizeri della rivista liberare e mazziniana L'Espero. Sul quaderno della giovane compaiono i nomi più importanti del Risorgimento genovese e italiano.

9. I periodici del Risorgimento nelle raccolte dell'Istituto Mazziniano, Mostra Storica sotto il patrocinio della Regione Liguria, Genova, Casa Mazzini, 31 maggio-29 luglio 1978. Saggi di storia del giornalismo in memoria di Leonida Balestreri, Genova, 1982. M. Milan, Giornali e periodici a Genova tra Ottocento $e$ Novecento, in D. Puncuch (a cura di), Storia della cultura ligure, "Atti della Società Ligure di Storia Patria», Nuova Serie, vol. XLV (CXIX), fasc. I, 2005, pp. 489-492.

10. Descrizione Di Genova e Del Genovesato, 3 volumi, Genova, Ferrando, 1846.

11. Lettera di Pio IX a G. B. Cevasco, 9 maggio 1856, Archivio Istituto Mazziniano Genova [infra AIMGe], n. 3382, Genova.

12. C. Sorba, Il melodramma della nazione, Bologna, Laterza, 2019, p. 70.

13. Lettera alla Giunta Comunale del 22 marzo 1851, AIMGe, n. 23454.

14. Da Genova opere recenti dello scultore Cevasco, «Rivista di Firenze: Bullettino delle Arti e del Disegno», vol. I, 1857, p. 236.

15. Ivi, p. 237.

16. E. Tonizzi, Genova nell'Ottocento, Cosenza, Rubbettino, 2013, pp. 115-120.

17. Lettera da Torino di C. Cavour a G. B. Cevasco, AIMGe, n. 3216.

18. R. Bottini, Emanuele Celesia, cit., pp. 130-131.

19. Lettera di Pio IX a G. B. Cevasco, 9 maggio 1856, AIMGe, n. 3382.

20. Si veda R. Bottini, Emanuele Celesia, cit., p. 149.

21. Lettera di G. B. Cevasco a V. Ricci, 22 gennaio 1855, AIMGe, n. 23453.

22. Lettera di G. Garibaldi, 6 dicembre 1854, AIMGe, n. 3268.

23. Lettera di G. Garibaldi a G. B. Cevasco, 16 agosto 1858, AIMGe, n. 3270.

24. Lettera da Torino di N. Bixio a G. B. Cevasco, 1864, AIMGe, n. 3171.

25. Lettera di G. B. Cevasco a R. Rubattino, 12 marzo 1876, AIMGe, n. 3180.

26. Lettera dell's maggio 1866 di G. B. Cevasco a G. Airenti, AIMGe, n. 3125.

27. Dopo l'Unificazione dell'Italia avvenuta il 17 marzo 1861, si assiste ancora ad un periodo di instabilità geopolitica ed economica. Vd. E. Tonizzi, Genova nell'Ottocento, cit., pp. 209-210.

28. A. M. Banti, Sublime madre nostra. La nazione italiana dal Risorgimento al fascismo, Bari, Laterza, 2012, p. 7.

29. Ivi, p. 9.

30. G. L. Mosse, La nazionalizzazione delle masse. Simbolismo politico e movimenti di massa in Germania (1812-1933), Bologna, Il Mulino, 1974.

31. G. Mazzini, Agli italiani, e specialmente agli operai italiani, in Id., Scritti editi ed inediti, vol. XXV, Imola, Galeati, 1916, pp. 10-11.

32. C. Strinati, Introduzione, in G. Mazzini, Filosofia della musica, Roma, La Lepre edizioni, 2019, p. 9.

33. Ivi, p. 10.

34. A. M. Banti, Sublime madre nostra, cit., p. 30.

35. C. Strinati, Introduzione, in G. Mazzini, Filosofia della musica, cit., p. 53.

36. Ivi, p. 11.

37. Ivi, p. 55.

38. Ivi, p. 20. 
39. Ivi, p. 24.

40. Ivi, p. 43.

41. Ibid.

42. F. Alizeri, Le feste genovesi per le faustissime nozze di S.A.R. Vittorio Emanuele Duca di Savoia con S.A.I. e R. Maria Adelaide Arciduchessa d'Austria, Genova, Arnaldo Forni Editore, 1984, p. 11.

\section{RIASSUNTI}

Nella prima parte del saggio si analizzano i sentimenti e gli atteggiamenti degli intellettuali del Risorgimento genovese animati inizialmente, grazie a Giuseppe Mazzini, da un forte entusiasmo rivoluzionario e repubblicano. Presto però si approda a un ritorno all'ordine e a un adeguamento a questa nuova Italia unita ma monarchica. Un secolo di sovranismo e di ritorno all'ordine segnano la Genova repubblicana che non dimentica però il suo passato e il suo apostolo rivoluzionario. Le arti e la musica accompagnano questa storia intellettuale di genovesi illustri che fecero della politica, della poesia, dell'arte, della scultura e della musica la loro bandiera unitaria, come mostra il pensiero mazziniano sulla musica, forza corale del popolo. In modo simile, Giovanni Battista Cevasco è impegnato nella scultura ma anche nelle istituzioni cittadine.

La première partie de l'essai analyse les sentiments et les attitudes des intellectuels du Risorgimento génois animés initialement, grâce à Giuseppe Mazzini, par un fort enthousiasme révolutionnaire et républicain, suivi par un retour à l'ordre et par une adaptation à cette nouvelle Italie unie mais monarchique. Un siècle de souveraineté et un retour à l'ordre marquent la Gênes républicaine qui n'oublie pas son passé et son apôtre révolutionnaire. Les arts et la musique accompagnent cette histoire intellectuelle d'illustres Génois qui ont fait de la politique, de la poésie, de l'art, de la sculpture et de la musique leur étendard unitaire, comme le montrent les réflexions de Mazzini sur la musique, force chorale du peuple. De même, dans le domaine des arts, on voit Giovanni Battista Cevasco s'engager dans la sculpture, mais aussi dans les institutions de la ville.

The first part of the essay analyzes the feelings and attitudes of the intellectuals of the Genoese Risorgimento, animated initially, thanks to Giuseppe Mazzini, by a strong revolutionary and republican enthusiasm. Soon, however, there was a "return to order" (ritorno all'ordine) and an adjustment to this new united but monarchical Italy. A century of sovereignty and a return to order marked the republican Genoa that did not forget its past and its revolutionary apostle. The arts and music accompany this intellectual history of illustrious Genoese who made of politics, poetry, art, sculpture and music their unitary banner, as shown by Mazzini's thought on music, the choral force of the people. In a similar way, Giovanni Battista Cevasco was engaged in sculpture but also in the city's institutions.

\section{INDICE}

Mots-clés : Risorgimento, république, arts

Keywords : Risorgimento, republic, arts

Parole chiave : Risorgimento, repubblica, arte 


\section{AUTORI}

MAURIZIA MIGLIORINI

Università di Genova

migma@unige.it

FRANCESCA GASTALDO

Università di Genova

CHIARA CAPOBIANCO

Università di Genova 